\title{
BEHAVIOR OF A SANDWICH BEAM SUBJECTED TO BENDING IN TERMS OF THE CORE'S SHAPE AND DENSITY
}

\begin{abstract}
Problem of the multi-layer sandwich beams, subjected to bending, is considered in this paper. The influence of the density and shape of the sandwich beam core on the beam behavior was studied. The two beam configurations were analyzed: the beam whose cross-section is constant along its length and the beam with periodically varying cross-section along the length. Each of the two configurations was considered for the two different core shapes - with the square and the circular holes. The diagrams of the beam deflection in terms of the number of the core unit cells are presented for the two beam configurations and the two core shapes. Based on results presented in this paper, one can conclude that in the given loading conditions, the core density, after reaching certain critical value, ceases to affect the bent sandwich beam behavior. Comparison of the two cores shapes leads to conclusion that, in the same loading conditions, the core with the square holes provides for the better sandwich beam's characteristics, i.e. such a beam has smaller deflection with respect to the beam with the core with the circular holes.

Keywords: Sandwich beam, bending, core shape, static analysis, optimization.
\end{abstract}

\section{Introduction}

Light multilayer constructions and sandwich structures were studied by numerous investigators: Allen [1] has set the fundamentals for design and analysis of the sandwich structures. In paper by Evans et al. [2] is presented a survey of investigations of the metal cellular systems' mechanical and thermal characteristics. Brittain et al. [3] have created a new method for manufacturing the micro plates with the truss core and they analyzed their mechanical properties. Wicks and Hutchinson [4] have shown that characteristics of the optimized sandwich plates with the truss core are superior to those of plates with the honeycomb core or the stringer-stiffened plates. Wicks and Hutchinson [5] have further extended the previous analysis to sandwich plates with truss core subjected to fracture straining and combination of the bending moment and transversal force, with restraining to buckling and plastic yielding. Valdevit et al. [6] have dealt with the structural optimization of the sandwich plates with the truss core. The multifunctional sandwich plates with prismatic and truss cores were analyzed from the similar point of view in works by Bujnak et al. [7] and Nikolic et al. [8]. The comparison of the two types of plates' performances to plates with honeycomb structure was performed. The estimates of the optimal plates' dimensions and the minimal mass of the sandwich plates with the prismatic and the truss cores were done.

Geometrically considered, these structures represent a result of periodical repetition of the basic cell in one, two, or three dimensions. The sandwich beam is an example of the periodical repetition of a unit cell in one direction (dimension) in order to obtain the multilayer or the sandwich structure.

Sandwich structures are found widely used in the aerospace, automobile and construction industry, as the highly resistant components, since they possess high stiffness and strength, as well as very good heat conductivity and low mass. Functionality of the sandwich beams, columns and other structural elements, can be understood if one analyzes the geometrical and mechanical factors affecting those elements' behavior under loading.

The sandwich beam is viewed as the multi-layer structure with the symmetrical cross-section. The assumption in this paper was that the sandwich structure was formed by the periodical repetition of a unit cell. Here is considered the influence of the unit cell's size, i.e. number of unit cells within structure, on static behavior of a sandwich beam subjected to bending. The static analysis of such a beam is done in this paper, namely the beam's bending stiffness and deflection are determined as a function of the number of the core unit cells, i.e. of the core's density. The

\footnotetext{
* ${ }^{1}$ Ruzica R. Nikolic, ${ }^{2}$ Jelena M. Djokovic, ${ }^{3}$ Jan Bujnak

${ }^{1}$ Faculty of Engineering, University of Kragujevac, Serbia, Research Center, University of Zilina, Slovakia

${ }^{2}$ Technical Faculty of Bor, University of Belgrade, Serbia

${ }^{3}$ Faculty of Civil Engineering, University of Zilina, Slovakia

E-mail: ruzicarnikolic@yahoo.com
} 
analyzed sandwich beam has length $L$; it is clamped at one end and free at the other where it is loaded by the transversal force $F$. The two considered configurations of the sandwich beams are shown in Figs. 1 and 2, each with two different core shapes, for the purpose of comparison of their influence on the beam's behavior in the given loading conditions.

\section{The considered problem definition}

In Fig. 1 is shown the sandwich beam with a cross section, which is constant along the beam's length $L$. The cross section consists of two flat plates, the beam's faces, upper and lower, of thickness $t_{f}$ and width $b$. The beam's core, of height $H_{C}$, is made of

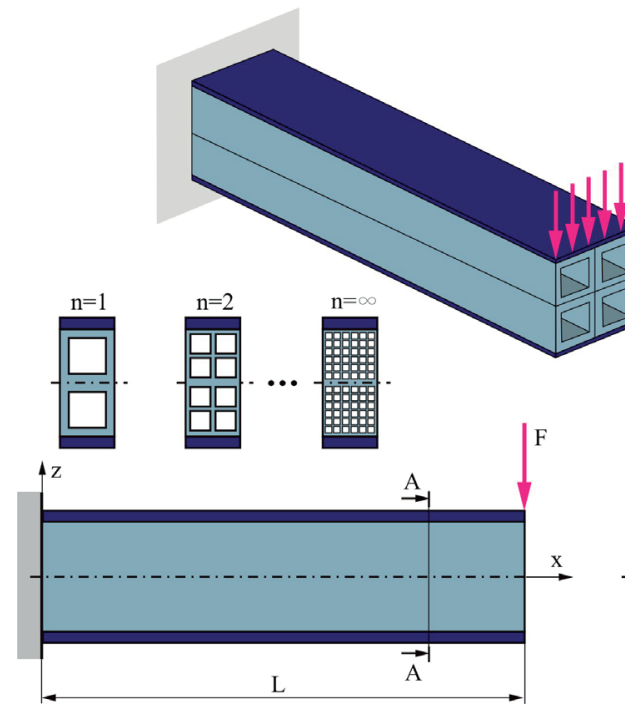

(a)

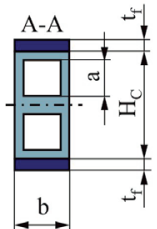

Fig. 1 Schematic presentation of the sandwich beams with the cross section constant along the length: (a) the core with square holes, (b) the core with circular holes

(b)

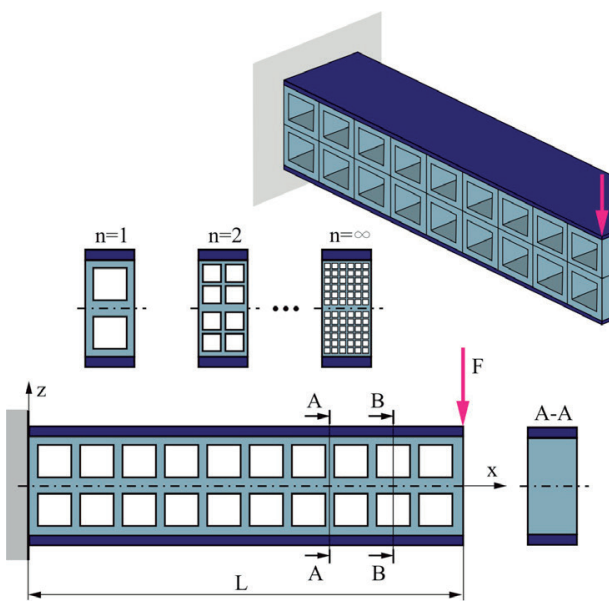

(a) a unit cell with two square holes of size $a$, shown in Fig. 1(a). This is a sandwich structure with $n=1$, where $n$ represents a number of unit cells, i.e. the core's density. As can be seen in Fig. 1(a), the structure with $n=2$ has eight square holes. If the structure could be built with sufficient (arbitrary) number of holes, $n$ could be practically limitless $(n=\infty)$. In Fig. 1(b) is shown a sandwich structure where the core consists of a unit cell with two circular holes of diameter $d$ and in this case is also $n=1$. The structure with $n=2$ has eight circular holes and so on, as far as the number of holes is sufficiently big, when it is taken that $n=\infty$.

In Fig. 2 is shown the sandwich beam with the cross section that is periodically changing along its length. In the A-A section, the beam has the solid cross section, while in the B-B section the cross section has the square or the circular holes. The upper and

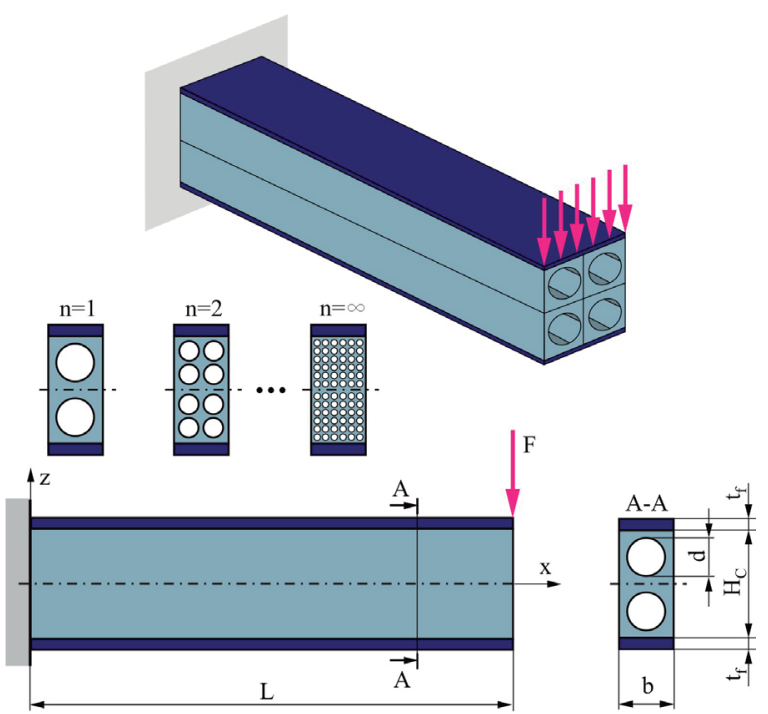

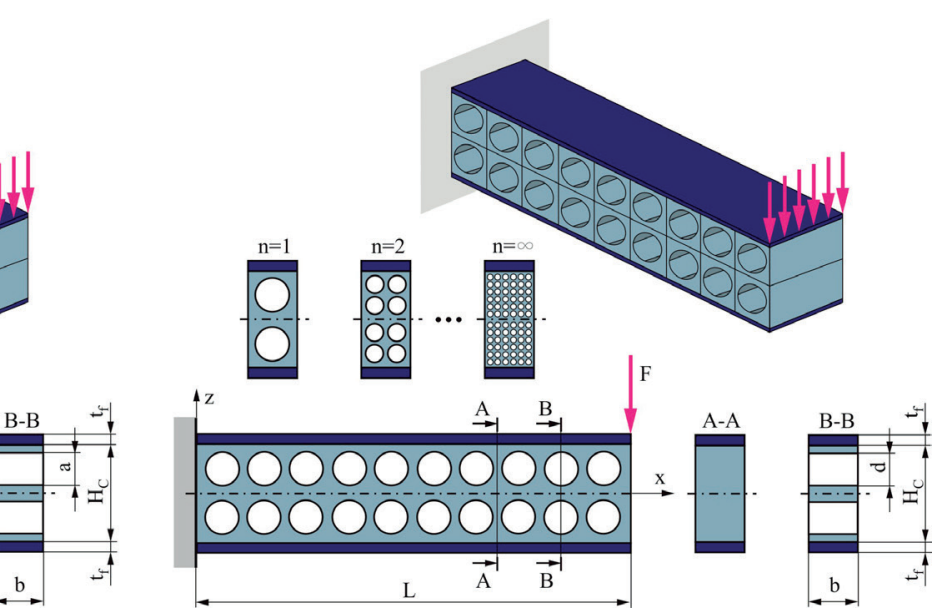

(b)

Fig. 2 Schematic presentation of the sandwich beams with the cross section that is variable along the length: (a) the core with square holes; (b) the core with circular holes 
the lower beam faces have the same thickness, $t_{f}$ while the beam's core height is $H_{C}$. The sandwich beams have the square holes of side $a$ and the circular holes of diameter $d$; the unit cell number is $n=1$ for both beams.

The faces and cores are made of different materials, thus the Young's elasticity modulus and the Poisson's ratio for the faces' material have subscript 1, i.e. $E_{1}$ and $v_{1}$, respectively and for the core material subscript 2, $E_{2}$ and $v_{2}$, respectively.

The differential equation of the beam's elastic (deflection) line reads:

$B^{(n)} \frac{d^{2} u(x)}{d x^{2}}=M(x)$,

where $B^{(n)}$ is the bending stiffness, $u(x)$ is the deflection and $M(x)$ is the bending moment for the cross section defined by coordinate $x$.

For the beam whose cross section is constant along its length, the bending stiffness is calculated as:

$B^{(n)}=\sum E_{i} I_{i}$,

while for the beam whose cross section is variable along its length the bending stiffness is calculated as:

$B^{(n)}=\left(\frac{1}{l} \int_{0}^{l} \frac{1}{B(x)} d x\right)^{-1}=l\left(\sum_{i} \frac{l_{i}}{B_{i}}\right)^{-1}$,

where $l$ is the period's length.

For the loading conditions of beams presented in Figs. 1 and 2 , the largest deflection is obtained at the beams' free ends, i.e. for $x=L$, and can be calculated as:

$u_{\max }=\frac{F L^{3}}{3 B^{(n)}}$.

For the beam configuration presented in Fig. 1(a) - the beam with the square cross section with the square holes, which does not vary along the beam's length, the bending stiffness in terms of the core's density is obtained as:

$$
\begin{aligned}
& B^{(n)}=\sum E_{i} I_{i}=2 E_{1} I_{1}+2 E_{2} I_{2}, \\
& I_{1}=\frac{1}{3} b\left[\left(t_{f}+\frac{H_{c}}{2}\right)^{3}-\left(\frac{H_{c}}{2}\right)^{3}\right], \\
& I_{2}=\frac{1}{3} b\left(\frac{H_{c}}{2}\right)^{3}-\frac{1}{3} \frac{a}{n} \sum_{m=1}^{n} n . \\
& .\left[\left(\frac{(2 m-1) H_{c}}{4 n}+\frac{a}{2 n}\right)^{3}-\left(\frac{(2 m-1) H_{c}}{4 n}-\frac{a}{2 n}\right)^{3}\right] .
\end{aligned}
$$

For the beam configuration presented in Fig. 1(b) - the beam with the square cross section with the circular holes, which does not vary along the beam's length, the bending stiffness in terms of the core's density is obtained as:

$$
\begin{aligned}
& B^{(n)}=\sum E_{i} I_{i}=2 E_{1} I_{1}+2 E_{2} I_{2}, \\
& I_{1}=\frac{1}{3} b\left[\left(t_{f}+\frac{H_{c}}{2}\right)^{3}-\left(\frac{H_{c}}{2}\right)^{3}\right], \\
& I_{2}=\frac{1}{3} b\left(\frac{H_{c}}{2}\right)^{3}-n \sum_{m=1}^{n} . \\
& \cdot\left[\frac{\pi}{2}\left(\frac{d}{2 n}\right)^{4}-\pi\left(\frac{(2 m-1) H_{c}}{4 n}\right)^{3}\left(\frac{d}{2 n}\right)^{2}\right] .
\end{aligned}
$$

For the beam configuration presented in Fig. 2(a) - the beam with the square cross section with the square holes, which is varying along the beam's length, the bending stiffness in terms of the core's density is obtained as:

$B^{(n)}=2 E_{1} I_{1}+B_{\text {periodic }}^{(n)}$,

$B_{\text {periodic }}^{(n)}=2 E_{2} \frac{I_{2} I_{3} H_{c}}{\left(H_{c}-a\right) I_{3}+2 a I_{2}}$,

$I_{1}=\frac{1}{3} b\left[\left(t_{f}+\frac{H_{c}}{2}\right)^{3}-\left(\frac{H_{c}}{2}\right)^{3}\right], I_{2}=\frac{1}{3} b\left(\frac{H_{c}}{2}\right)^{3}$,

$I_{3}=\frac{1}{3} b\left(\frac{H_{c}}{2}\right)^{3}-\frac{1}{3} \frac{a}{n} \sum_{m=1}^{n} n$

$\left[\left(\frac{(2 m-1) H_{c}}{4 n}+\frac{a}{2 n}\right)^{3}-\left(\frac{(2 m-1) H_{c}}{4 n}-\frac{a}{2 n}\right)^{3}\right]$.

For the beam configuration presented in Fig. 2(b) - the beam with the square cross section with the circular holes, which is varying along the beam's length, the bending stiffness in terms of the core's density is obtained as:

$B^{(n)}=2 E_{1} I_{1}+B_{\text {periodic }}^{(n)}$

$B_{\text {periodic }}^{(n)}=\frac{2 E_{2}}{H_{c}}\left\{\int_{0}^{\frac{H_{c}-d}{4 n}} d x /\left(\frac{b H_{c}^{2}}{24}\right) \int_{0}^{\frac{H_{c}-d}{4 n}} d x /\left\{\frac{b H_{c}^{2}}{24}-\frac{n b}{3}\right.\right.$.

$\sum_{m=1}^{n}\left[\left(\frac{(2 m-1) H_{c}}{4 n}+\sqrt{\left(\frac{d}{2 n}\right)^{2}-\left(x-\frac{(2 m-1) H_{c}}{4 n}\right)}\right)^{3}\right.$

$\left.\left.-\left(\frac{(2 m-1) H_{c}}{4 n}-\sqrt{\left(\frac{d}{2 n}\right)^{2}-\left(x-\frac{(2 m-1) H_{c}}{4 n}\right)}\right)^{3}\right]\right\}$

$\left.+\int_{\frac{H_{c}+d}{4 n}}^{\frac{H_{c}}{2 n}} d x /\left(\frac{b H_{c}^{2}}{24}\right)\right\}^{-1}$

$I_{1}=\frac{1}{3} b\left[\left(t_{f}+\frac{H_{c}}{2}\right)^{3}-\left(\frac{H_{c}}{2}\right)^{2}\right]$.

\section{Results and discussion}

In Fig. 3 is presented the variation of the beam's free end's deflection in terms of the core's density for the beam whose cross section does not vary along its length, for the two shapes of the core and the same loading conditions. Results are obtained based on equations (4) to (6) by application of the Mathematica ${ }^{\circledR}$ symbolic programming routine. 


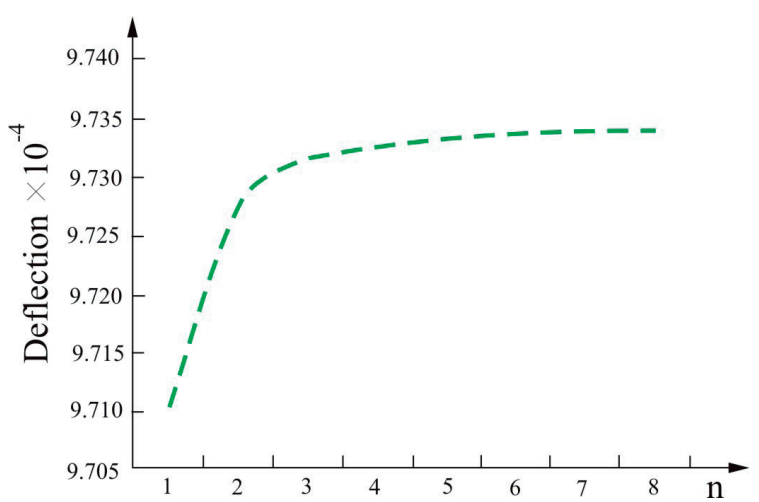

(a)

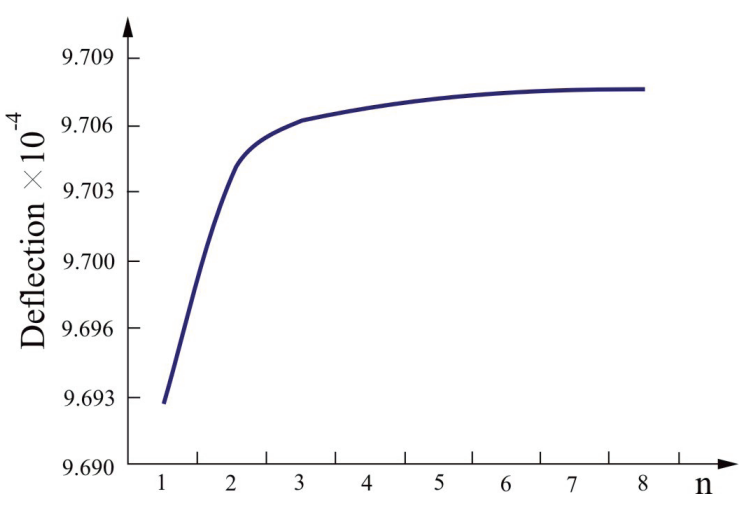

(b)

Fig. 3 Deflection of the free end of the bent sandwich beam with the constant cross section along in terms of the core's density $n$ : (a) the core with square holes; (b) the core with circular holes

From Fig. 3, one can notice that the value of the beam's deflection increases with the core density, while after reaching a value of approximately $n=5$ the deflection almost remains constant.

In Fig. 4 is presented the variation of the beam's free end's deflection in terms of the core's density for the beam whose cross section varies periodically along its length, for the two shapes of the core and the same loading conditions. Results are obtained based on equations (4), (7) and (8) by application of the Mathematica $^{\circledR}$ symbolic programming routine.

From Fig. 4 one can see that similarly as for the first beams' configurations, when the cross section is constant, the value of the beam's deflection increases with the core density, while after becoming approximately $n=5$ the deflection almost remains constant. So, the conclusion is that, regardless of the configuration, after that critical value, the increase of the core density does not significantly influence the sandwich beam's behavior, if at all.

In Fig. 5 is presented variation of the bent sandwich beam free end's deflection in terms of the core density, for the same configuration and for the two shapes of the core.

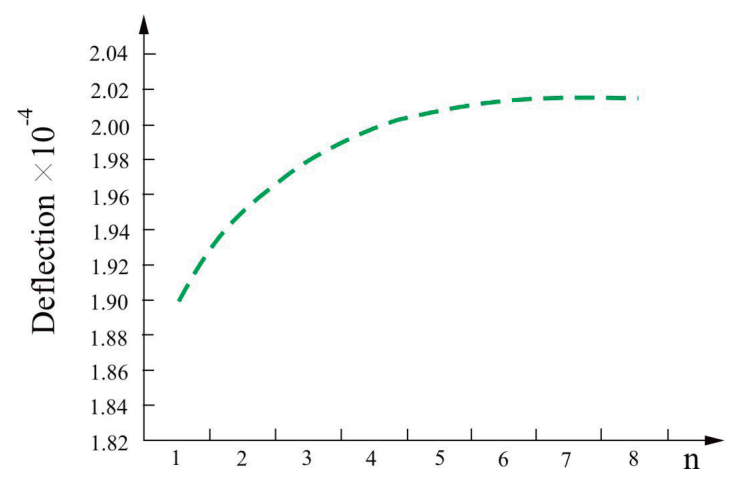

(a)

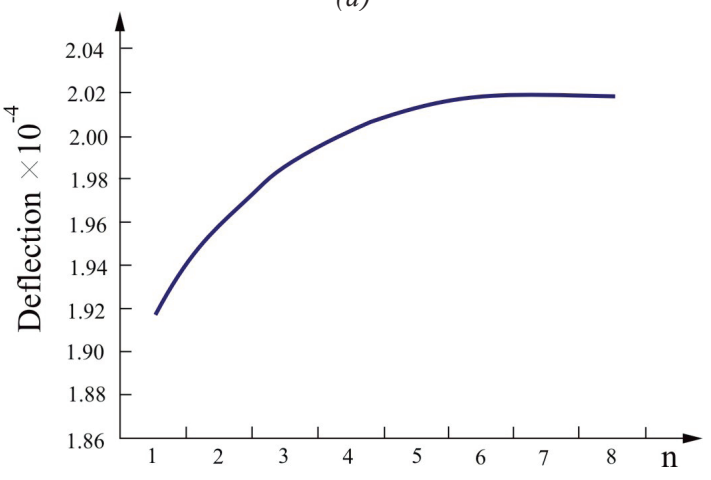

(b)

Fig. 4 Deflection of the free end of the bent sandwich beam with the periodically varying cross section in terms of the core density $n$ : (a) the core with square holes; (b) the core with circular holes

From Fig. 5 could be seen that, for the same loading conditions, the beam with the square holes has smaller deflection than the beam with the circular holes.

If the two configurations were compared with respect to the value of the free end's deflection, one could conclude that the beam with the periodically varying cross section is somewhat better than the beam whose cross section is constant along its length, under the same loading conditions.

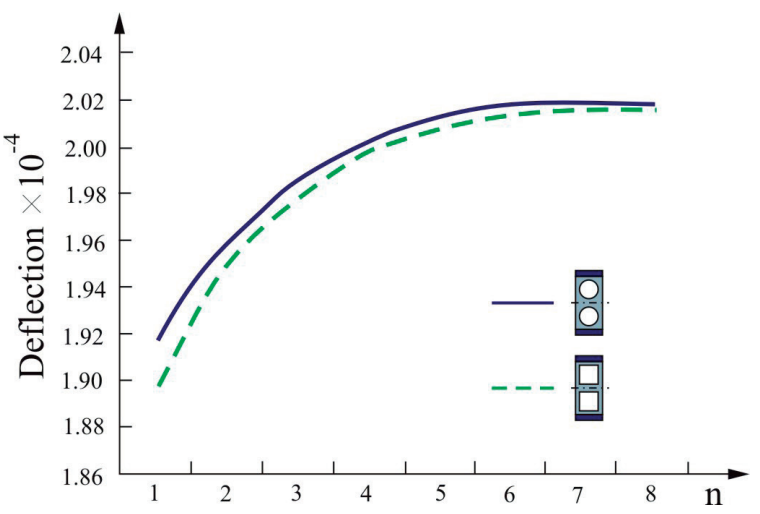

Fig. 5 Deflection of the free end of the bent sandwich beam with the cross section periodically varying along its length in terms of the core density $n$ for the two shapes of the core 


\section{Conclusion}

The influence of the shape and density of the core on behavior of the bent sandwich beam was analyzed in this paper. The sandwich beam was considered as the multi-layered structure with the symmetric cross-section. It was assumed that the sandwich structure is formed by periodical repetition of the core's unit cell. The attention was devoted to influence of the size of the unit cell, i.e. the number of unit cells on behavior of the sandwich beam subjected to bending. The static analysis of that behavior included determination of the sandwich beam free end's deflection in terms of the beam's core density.

The two configurations of the sandwich beams were considered: with the cross-section that is constant and that is varying along the beams length, with the two different shapes of the core: with the square and the circular holes.

The diagrams of the beams' free end's deflection were obtained for all the configurational combinations by application of the Mathematica ${ }^{\circledR}$ symbolic programming routine. Based on obtained results the conclusion was drawn that the core density influences the bent sandwich beam behavior up to a certain critical value (in cases considered in this paper that value was $n=5$ ), after which that influence is practically negligible. The other conclusion is that, in the given loading conditions, the beam whose cross section is periodically varying along its length is somewhat better than the beam whose cross section is constant.

\section{Acknowledgement}

Parts of this research were financially supported by the Ministry of Education, Science and Technological Development of Republic of Serbia through Grants ON174001, ON174004 and TR32036 and by the European regional development fund and Slovak state budget by the project "Research Center of the University of Zilina” - ITMS 26220220183.

\section{References}

[1] ALLEN, H. G.: Analysis and Design of Structural Sandwich Panels, Pergamon Press, 1969.

[2] EVANS, A. G., HUTCHINSON, J. W., ASHBY, M. F.: Multifunctionality of Cellular Metal Systems, Progress in Materials Science, vol. 43(3), 1998, pp. 171-221.

[3] BRITTAIN S. T., SUGIMURA, Y., SCHUElleR, J. A., EVANS, A. G., WHITESIDES, G. M.: Fabrication and Mechanical Performance of a Mesoscale, Space-Filling Truss Systems, J. of Microelectromechanical Systems, 10, 2001, pp. 113-120.

[4] WICKS, N. HUTCHINSON, J. W.: Optimal Truss Plates, Intern. J. of Solids and Structures, col. 38, 2001, pp. 5165-5183.

[5] WICKS, N., HUTCHINSON, J. W.: Performance of Sandwich Plates with Truss Cores, Mechanics of Materials, vol. 36, 2004, pp. 739-751.

[6] VALDEVIT, L., HUTCHINSON, J. W., EVANS, A. G.: Structurally Optimized Sandwich Panels with Prismatic Cores, Intern. J. of Solids and Structures, vol. 41, 2004, pp. 5105-5124.

[7] BUJNAK, J., DJOKOVIC, J. M., NIKOLIC, R. R.: Optimal Plates with Prismatic Cores, Roczniki Inzynerii Budowlanej - Annals of Civil Engineering, vol. 13, 2013, pp. 13-20.

[8] NIKOLIC, R. R., DJOKOVIC, J. M., BUJNAK, J.: Optimization of Sandwich Plates with Truss Cores, Roczniki Inzynierii Budowlanej - Annals of Civil Engineering, vol. 12, 2012, pp. 5-11. 